\title{
Historically low mitochondrial DNA diversity in koalas (Phascolarctos cinereus)
}

\author{
Kyriakos Tsangaras', María C Ávila-Arcos², Yasuko Ishida ${ }^{3}$, Kristofer M Helgen ${ }^{4}$, Alfred L Roca ${ }^{3}$
} and Alex D Greenwood ${ }^{1 *}$

\begin{abstract}
Background: The koala (Phascolarctos cinereus) is an arboreal marsupial that was historically widespread across eastern Australia until the end of the $19^{\text {th }}$ century when it suffered a steep population decline. Hunting for the fur trade, habitat conversion, and disease contributed to a precipitous reduction in koala population size during the late 1800s and early 1900s. To examine the effects of these reductions in population size on koala genetic diversity, we sequenced part of the hypervariable region of mitochondrial DNA (mtDNA) in koala museum specimens collected in the $19^{\text {th }}$ and $20^{\text {th }}$ centuries, hypothesizing that the historical samples would exhibit greater genetic diversity.

Results: The mtDNA haplotypes present in historical museum samples were identical to haplotypes found in modern koala populations, and no novel haplotypes were detected. Rarefaction analyses suggested that the mtDNA genetic diversity present in the museum samples was similar to that of modern koalas.

Conclusions: Low mtDNA diversity may have been present in koala populations prior to recent population declines. When considering management strategies, low genetic diversity of the mtDNA hypervariable region may not indicate recent inbreeding or founder events but may reflect an older historical pattern for koalas.
\end{abstract}

\section{Background}

Though distributed widely across eastern Australia, the koala (Phascolarctos cinereus) was apparently a rare animal at the time of the first European settlement of Australia. Many of the relatively large mammals of eastern Australia (such as kangaroos, possums, quolls, and the dingo) were first documented by Europeans almost immediately after the settlement of modern-day Sydney in 1788, but the first European sighting of a koala was not reported until 1798. The first live specimen was secured and brought to Sydney in 1803, and very few detailed accounts of the animal appeared in the decade that followed. Remarkably, it was not until 1816, nearly three decades after the arrival of Britain's First Fleet to Botany Bay, that the koala was studied in sufficient detail to be given its original scientific name, Lipurus cinereus [1]. Most students of the koala have attributed the rarity of the animal in the late eighteenth and nineteenth centuries to the impacts of hunting by Aboriginal Australians. John Gould,

\footnotetext{
* Correspondence: greenwood@izw-berlin.de

'Leibniz-Institute for Zoo and Wildlife Research, Berlin 10315, Germany

Full list of author information is available at the end of the article
}

among the most notable nineteenth-century naturalists working in Australia, discussed the rarity of the koala and the impacts of Aboriginal hunting upon it, and speculated that it was likely to decline to extinction. Instead, hunting pressure diminished during much of the $19^{\text {th }}$ century, and koala numbers are believed to have risen drastically, such that the koala was apparently a common animal by the middle to late 1800s across much of eastern Australia [1].

Soon after their numbers increased, koala fur became a highly valued commodity, and the koala was hunted with tremendous intensity for the international fur trade. Throughout the late 1800s and early 1900s, millions of koalas are thought to have been killed for their fur every year. Koala declines from intensive hunting during this period were also exacerbated by habitat conversion leading to habitat loss and range fragmentation (the great majority of relevant koala habitat present at European settlement had been cleared by the twentieth century) [1]. Many historical reports also describe mass declines of koalas from various epidemic diseases, dating back to the 1880s [1]. By the 1930s, from the combined effects of hunting, habitat conversion, and disease, koala populations had
Ciomed Central

(c) 2012 Tsangaras et al.; licensee BioMed Central Ltd. This is an Open Access article distributed under the terms of the Creative Commons Attribution License (http://creativecommons.org/licenses/by/2.0), which permits unrestricted use, distribution, and reproduction in any medium, provided the original work is properly cited. 
been extirpated across much of southern Australia and were greatly diminished in the northern range of the species [2,3].

A severe decrease in population size has been shown to lead to loss of genetic diversity in other species [4]. For example, the US grey wolf (Canis lupus) lost diversity due to widespread extirpation of local populations. Leonard et al. [5] demonstrated using modern and museum samples that the museum wolf samples had twice the diversity of modern conspecifics. The decrease in numbers of koalas would likewise suggest that the species may exhibit reduced genetic diversity due to population bottlenecks. Reduced genetic diversity is associated with a variety of potential threats in affected populations, such as a decrease in population fitness due to inbreeding, expression of deleterious recessive alleles, reduced disease resistance, and lack of heterosis $[2,6]$.

Several molecular markers have been used to examine the genetic variability of modern Australian koala populations; these have shown that genetic diversity within and gene flow between koala populations is low [3,7-9]. Houlden et al. [10] examined 860 bp of mtDNA hypervariable region in 208 koalas from 16 populations throughout Australia. Koala mtDNA haplotype diversity within populations averaged $\mathrm{H}=0.18$, which was only one-fifth the level of diversity detected in an Australian omnivore, the greater bilby (Macrotis lagotis). Genetic differentiation among the koala populations of Queensland and New South Wales was found to be high, while koala populations from Victoria were little differentiated [10], presumably due to their recent descent from few individuals from the French and Philip Islands. Fowler et al. [11] examined $670 \mathrm{bp}$ of the same mtDNA hypervariable region studied by Houlden et al. [10] from 96 koalas in five southeastern Queensland populations. Haplotype diversity within populations was estimated as $\mathrm{H}=0.37$, higher than Houlden et al. [10] but still much lower than bilby diversity.

The historical DNA diversity present among koalas can be examined using museum specimens, as mtDNA analyses from museum samples have been reported from many species including Australian wildlife [12-14]. We hypothesized that, compared to modern koalas, there would be greater genetic diversity in the museum specimens, particularly those collected in the 1800s and early 1900 s, before (or at the beginning of) the most recent prolonged historical population decline. Using ancient DNA approaches, the diversity of historical haplotypes in the museum koalas was compared to the diversity present in modern koalas.

\section{Results}

The koala has an 860 bp hypervariable mtDNA region that is adenine and thymine rich. Primer sets were designed to amplify fragments of $73 \mathrm{bp}$ and $112 \mathrm{bp}$ (excluding primer lengths) of this mtDNA region and were tested on a modern koala DNA dilution series. The primers were designed to target small amplicons to accommodate the potential degradation that has often been observed in archival museum samples. We attempted DNA extractions and PCR on 29 different museum samples originally collected from the 1870 s to the 1980 s (Table 1). Across the 29 samples, we were able to amplify and sequence both of the mtDNA fragments from 15 samples representing 14 individual koalas (Table 1). An additional 1 sample was successfully amplified and sequenced for only the $73 \mathrm{bp}$ mtDNA fragment, potentially indicating that the DNA from that sample was more highly degraded. Thirteen samples failed to amplify for both of the mtDNA fragments. The amplicons were sequenced using two approaches: GS FLX and Sanger direct sequencing (see Materials and Methods for details). The seven GS FLX analyzed samples displayed minimal levels of the damage usually associated with ancient DNA (Figure 1) and the results were found to be comparable between the two approaches. Thus, direct Sanger sequencing of amplicons appeared to be an appropriate strategy for these museum samples.

The sequences from the two adjacent mtDNA segments indicated that 4 unique haplotypes were present across the 14 koalas (Table 1, Figure 2A). Each of the four museum sample mtDNA haplotypes was identical to one of the previously reported modern haplotypes identified by Houlden et al. [10], and Fowler et al. [11]. No novel mtDNA haplotype was detected among the historical samples.

Minimum spanning networks were generated to demonstrate the relationships across the mtDNA haplotypes (Figure 2A). The koala archival samples carried haplotypes identical to modern haplotypes $\mathrm{K} 1, \mathrm{~K} 3, \mathrm{~K} 4$, and K5. Only one or few mutations separated these haplotypes $[8,10,11]$. All but one of the sequenced museum samples were collected in northern Australia. The haplotypes observed among northern Australian museum samples (K3, K4 and K5) were also found among modern samples collected in northern Australia only (K3, K5), or were found in both northern and southern modern koalas (K4) (Figure 2). Only one of the museum samples from southern Australia was successfully sequenced. The haplotype for this koala (Pci-AM M12482; Table 1) was identical to a haplotype characteristic of modern southern koalas (haplotype K1, Figure 2). Thus for the koala museum specimens, the geographic distribution of haplotypes proved consistent with those of modern koalas.

The number of museum sample sequences that we were able to obtain was lower than the number of modern samples that have previously been sequenced. Rarefaction analysis was used to estimate expected haplotype 
Table 1 Koala samples and sequencing information

\begin{tabular}{|c|c|c|c|c|c|}
\hline Koala sample origins & Designation/ museum no. & Collection year & Amplified mtDNA & Haplotype & Sequencing method \\
\hline \multicolumn{6}{|l|}{ Northern Australian Samples } \\
\hline Bohusläns Museum & Pci-um3435 & 1891 & $\otimes$ & K3 & GS FLX/ Sanger \\
\hline \multirow[t]{2}{*}{ Goteborg Museum } & Pci-collan18193 & $1870-1891$ & $\square$ & & \\
\hline & Pci-maex1738 & $1870-1891$ & $\otimes$ & K5 & GS FLX \\
\hline Kansas University Museum & Pci-159224 & $1980 \mathrm{~s}$ & $\nabla$ & K4 & GS FLX \\
\hline \multirow[t]{2}{*}{ Museum of Comparative Zoology } & PCi-MCZ 12454* & 1904 & $\otimes$ & K4 & GS FLX/ Sanger \\
\hline & Pci-MCZ 8574* & 1904 & $\otimes$ & & GS FLX \\
\hline \multirow[t]{2}{*}{ Museum of Victoria } & Pci-c2831 & 1923 & $\otimes$ & K4 & GS FLX/ Sanger \\
\hline & Pci-c2832 & 1923 & $\square$ & & \\
\hline \multirow[t]{10}{*}{ Queensland Museum } & Pci-QM J6480 & 1938 & $\otimes$ & K5 & GS FLX/ Sanger \\
\hline & Pci-QM J2377 & 1915 & $\square$ & & \\
\hline & Pci-QM J7209 & 1945 & $\otimes$ & K5 & Sanger \\
\hline & Pci-QM J8353 & 1952 & $\square$ & & \\
\hline & Pci-QM JM1875 & $1960 \mathrm{~s}$ & $\otimes$ & K4 & Sanger \\
\hline & Pci-QM JM64 & 1973 & $\otimes$ & K4 & Sanger \\
\hline & Pci-424 & $1970-1980 s$ & $\otimes$ & K5 & Sanger \\
\hline & Pci-6121 & $1970-1980$ s & $\otimes$ & K5 & Sanger \\
\hline & Pci-7463 & $1970-1980 s$ & $\otimes$ & K5 & Sanger \\
\hline & Pci-7625 & $1970-1980$ s & $\otimes$ & K5 & Sanger \\
\hline Royal Ontario Museum & Pci-9111010180 & 1891 & $\square$ & & \\
\hline Stockholm Museum & Pci-582119 & 1911 & 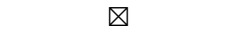 & K4 & GS FLX \\
\hline \multirow[t]{2}{*}{ U of Michigan Museum of Zoology } & Pci-122553 & 1966 & $\square$ & & \\
\hline & Pci-124673 & 1977 & $\square$ & & \\
\hline \multicolumn{6}{|l|}{ Southern Australian Samples } \\
\hline \multicolumn{6}{|l|}{ New South Wales } \\
\hline \multirow[t]{6}{*}{ Australian Museum } & PCi-AM M17311 & 1883 & $\square$ & & \\
\hline & PCi-AM M17299 & 1883 & $\square$ & & \\
\hline & PCi-AM M17300 & 1883 & $\square$ & & \\
\hline & PCi-AM B4593 & 1884 & $\square$ & & \\
\hline & PCi-AM M1461 & 1899 & $\square$ & & \\
\hline & Pci-AM M12482 & 1971 & $\otimes$ & K1 & Sanger \\
\hline \multicolumn{6}{|l|}{ Victoria } \\
\hline Australian Museum & Pci-AM M4841 & 1930 & $\square$ & & \\
\hline
\end{tabular}

$\mathrm{x}$ Indicates successful amplification and sequencing of sample mtDNA.

* Indicates that the two samples represent the same individual.

${ }^{\text {a }}$ Amplification and sequencing of a single fragment.

diversity of a modern sample set of the same size as the museum sample set. Using the modern DNA data, rarefaction analysis suggested that if 14 modern koalas had been sampled at random, 4 haplotypes would be identified, the same number of haplotypes that we were able to identify from our 14 museum samples (Figure 3A). This suggested that ancient koalas carried mtDNA diversity similar to that present in modern koala populations. Rarefaction analysis of the modern sample set was also plotted to evaluate whether the sequencing of additional museum specimens would be likely to identify a larger number of mtDNA haplotypes (Figure 3A). The analysis suggested that even a 2 -fold increase in museum samples would have identified only a single additional haplotype (Figure 3A). An additional analysis, separating the 7 oldest samples and seven youngest into two sets, predicted no differences in the expected number of retrievable haplotypes between the two sets (not shown).

Rarefaction analysis was also performed using a conjectural dataset in which the number of haplotypes of 


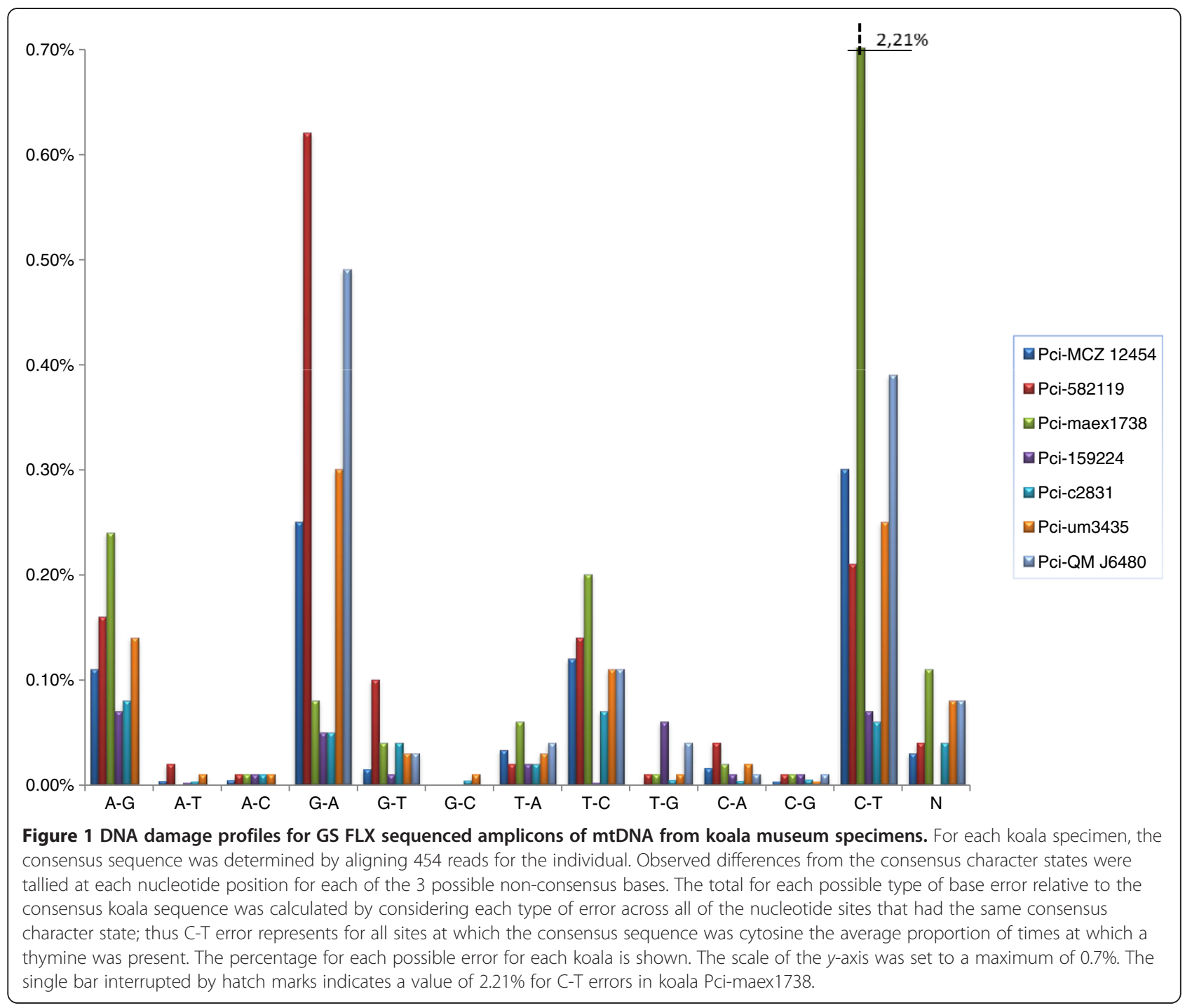

mtDNA was twice that actually observed e.g., 8 rather than the actual 4 haplotypes present across 14 samples. This conjectural dataset was meant to illustrate the initial hypothesis that mtDNA diversity would be higher in museum than in modern samples, as had been found in the greater bilby and grey wolf [5]. The results are shown in Figure 3B. As koala sample size increases, the number of haplotypes increases for modern and museum specimens, with the curve based on actual museum specimen results following the same trajectory as the curve followed by modern samples. By contrast, the conjectural diversity curve, postulating twice the diversity in the past relative to the present, had a different trajectory, with even a sample set comprised of a much smaller number of koalas yielding more haplotypes than actually observed (Figure 3B). The conjectural curve follows a much higher trajectory than actual modern or museum data sets. Thus the low number of historical haplotypes likely reflects limited haplotype diversity in the museum koalas equivalent to that in the modern samples, rather than the effects of limited sampling.

\section{Discussion}

Our study represents the first analysis of koala mtDNA to incorporate museum specimens. Sixteen of twentynine samples amplified for mtDNA; these ranged in year of collection from the late 1800s to the 1980s. Previous surveys of koalas have revealed that mtDNA diversity is quite low in modern populations [11]. We hypothesized that there would be greater diversity in the museum samples, particularly those from the 1800 s and early 1900 s, as compared to modern samples, expecting that genetic diversity would be greater prior to the severe population reductions that occurred from the late 1890s through the early $20^{\text {th }}$ century. Sequencing of $201 \mathrm{bp}$ of the mtDNA hypervariable region from 14 koalas yielded 

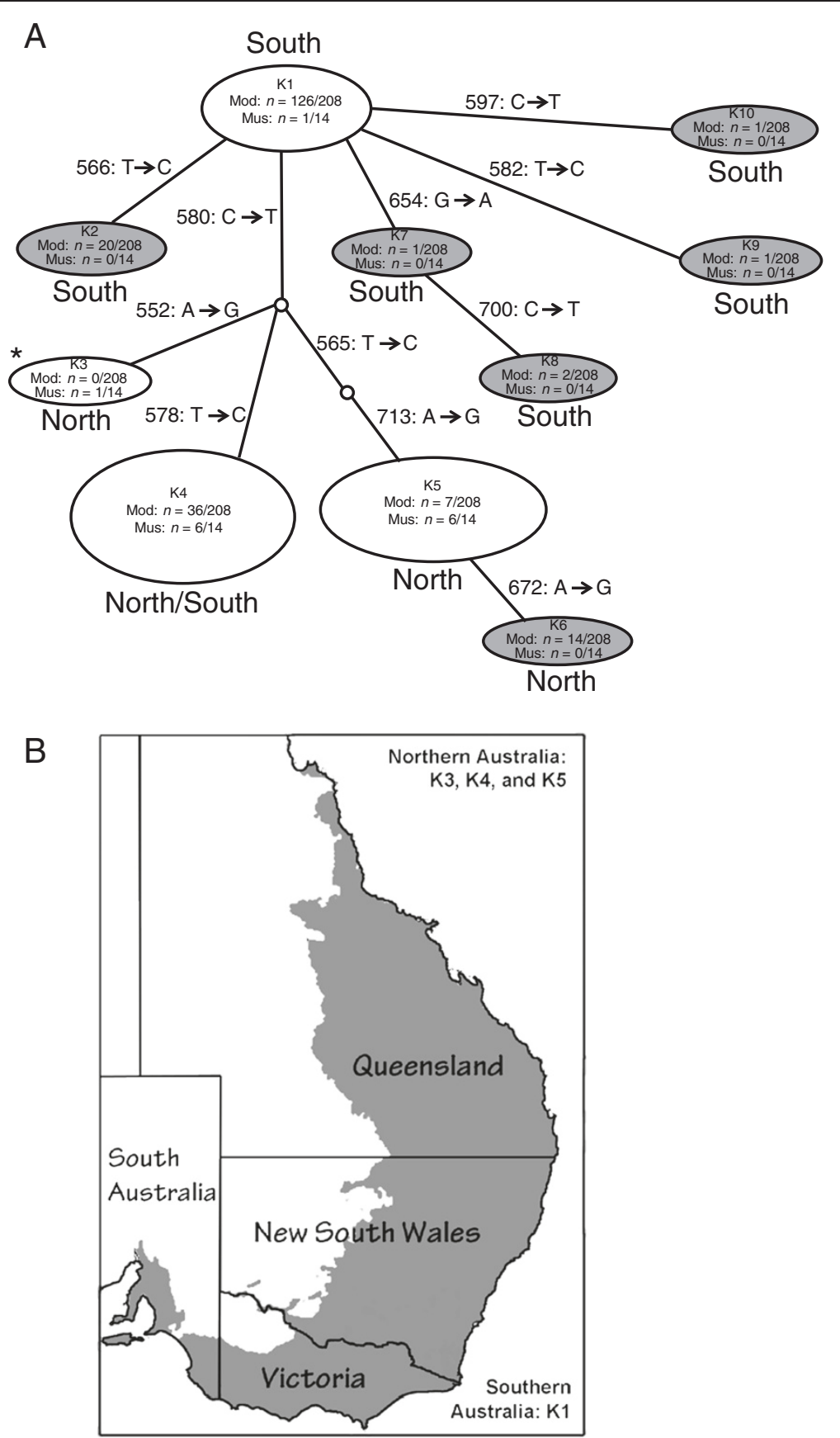

Figure 2 Panel A shows a minimum spanning network indicating the number of nucleotide differences detected among koala mtDNA hypervariable region haplotypes. Identical sequences were grouped together as a single unique haplotype. Unshaded nodes indicate haplotype sequences found in both museum and modern samples. Shaded nodes represent sequences detected only in modern samples. The position of the changes relative to the koala reference mtDNA genome (AJ005852) is indicated for each variant site by an arrow e.g. K5 differs from K6 by an A-G change at position 672 where K5 has the reference base adenine and K6 has a change to guanine at that position. Numbers of samples in which each haplotype was detected are indicated for modern ("Mod") and museum ("Mus") samples. For haplotype K3 (asterisk), identified by Fowler et al. [11], the number of modern koalas carrying the haplotype was not reported. The geographic distribution of each haplotype (northern or southern Australia, or both) is indicated. Panel B shows the approximate current range of koalas (shaded) in Australia. The historical haplotypes identified are indicated inside the parentheses. The map image was adapted from map images provided by the Australian Government, www.ga.gov.au. 


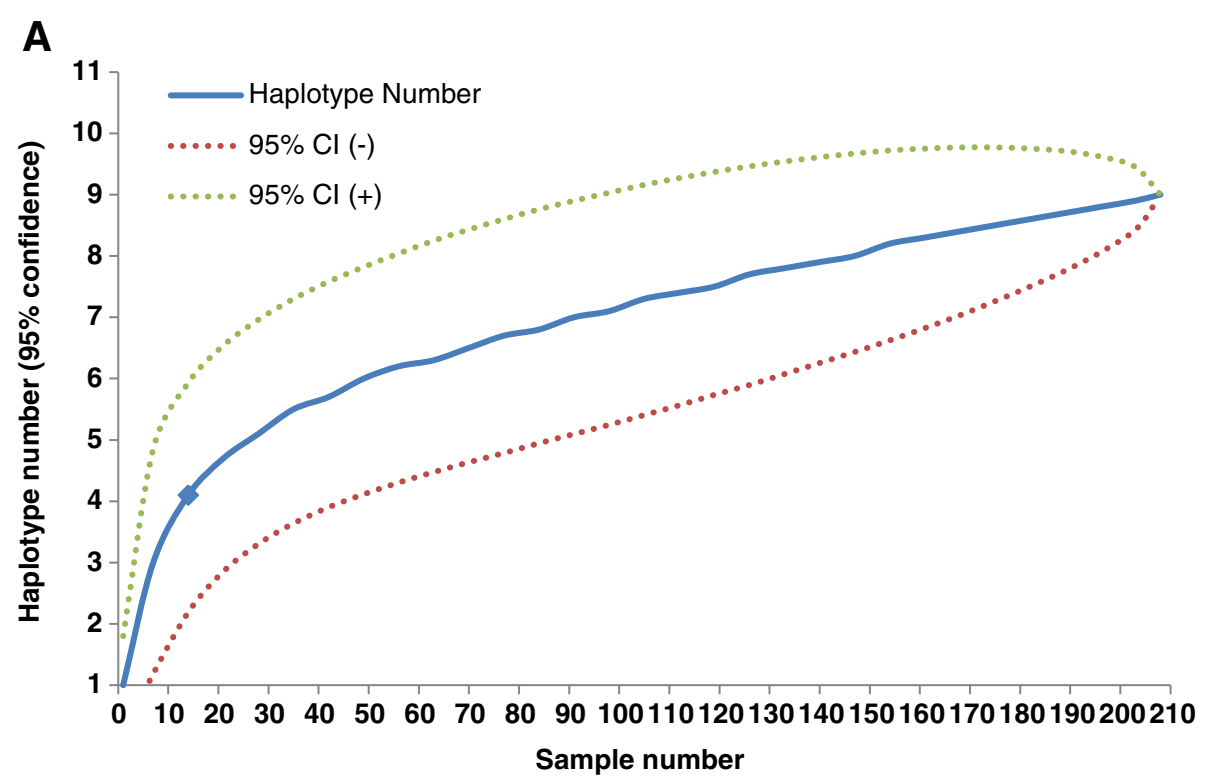

B

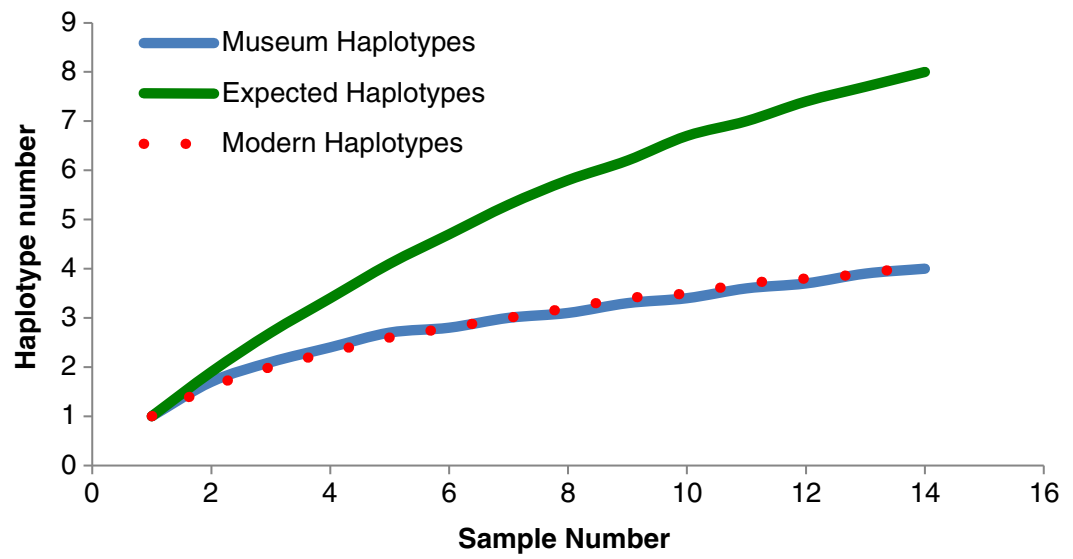

Figure 3 Rarefaction analyses of modern and ancient koala mtDNA haplotypes. Panel A shows the expected number of haplotypes based on random sampling of the modern koala dataset. Based on the Houlden et al. [8] dataset, one would expect that a random sample of 14 modern koalas would carry 4 unique haplotypes. The set of 14 museum samples for which mtDNA could be sequenced also carried 4 unique haplotypes (blue diamond), completely consistent with expectations based on modern mtDNA diversity. Panel B models mtDNA haplotype data based on random sampling of modern koalas, ancient koalas and a conjectural dataset. The solid blue curve represents the rarefied number of mtDNA haplotypes for modern koalas, showing the number of unique mtDNA haplotypes expected given sample sizes of up to 14 . The overlapping red dotted line indicates the number of mtDNA haplotypes for museum koalas. The overlap between curves representing modern and ancient koalas indicates that koala mtDNA diversity was not substantially higher in the museum samples than the modern samples. The green solid line represents rarefaction analysis of a conjectural population with twice the number of mtDNA haplotypes as modern koalas. The curve for the conjectural dataset deviates substantially from the curves drawn from modern and museum data, even at small sample sizes.

four haplotypes, all of which were present in modern koalas (Table 1 and Figure 2). The lack of novel mtDNA haplotypes in archival northern Australian specimens collected from a variety of populations and time points suggested that limited mtDNA variation was present in northern koalas before the known historical population reduction, which lowered koala numbers in northern Australia from approximately 1 million animals to ten thousand by 1927 [11].
Koala populations declined drastically across Australia in the early $20^{\text {th }}$ century, yet our museum sample data did not reveal that diversity was higher before the bottleneck. Rarefaction analysis indicated that these results were not due to unequal modern and museum sample sizes (Figure 3). Doubling the museum sample size can be predicted to have yielded only one additional unique haplotype. If museum koalas had greater mtDNA diversity, then more haplotypes should have 
been detected in the museum sequences, as occurred in ancient DNA studies of other species such as the US grey wolf [5]. However, this was not the case and we concluded that our results are not an artifact of the small sample sizes.

This provides an indication that older events may have been more important in shaping modern genetic diversity in koalas. Late Pleistocene extinctions in Australia, attributed to the impacts of Aboriginal arrival, climate change, or their combination, resulted in the extinction of most of the continent's larger animals (megafaunal marsupials, birds, and reptiles), as well as the extinction or decline of some medium-sized species, including koalas [15]. Prior to this extinction event, two koala species (both the modern $P$. cinereus and the larger $P$. stirtoni) co-existed in Australia, and the modern koala had a wider distribution that extended to south-western Western Australia [16,17]. The extinction of one koala species and the reduction of the geographic range of the living koala since the Pleistocene provide strong indications of Late Pleistocene koala declines, which in addition to reducing the taxonomic diversity of koalas, would also have likely impacted the genetic diversity of $P$. cinereus. Further, if the rarity of koalas at the time of European arrival to Australia provides an indication of a historical baseline for koala abundance under the impacts of Aboriginal hunting, it can be hypothesized that the limited genetic diversity seen in modern koalas might reflect a long history of relatively low population numbers throughout the range of koalas during the Late Pleistocene and Holocene, another demographic factor that may have strongly limited koala genetic diversity in recent millennia.

Historically low mitochondrial diversity is not unique to Australian koalas. Among Australian marsupials, low mtDNA diversity has also been reported in the Tasmanian devil (Sarcophillus harrisii). A recent study by Miller et al. [14] that compared modern Tasmanian devil mtDNA diversity with historical diversity based on 175 modern samples and six museum specimens collected between 1870 and 1910 revealed five historical haplogroups. Four were identical to haplogroups in modern Tasmanian devils and only one was unique to the historical samples. The authors concluded that Tasmanian devil mitochondrial diversity has generally been low over the past century even though the population had started to decline more recently due to disease [18]. Similarly, the extinct thylacine (Thylacinus cynocephalus) demonstrated low genetic diversity, which may have been a consequence of the separation of Tasmania from mainland Australia $[19,20]$. In non-Australian fauna, the Iberian lynx (Lynx pardinus) has maintained extremely low genetic diversity for at least 50,000 years [21]. Thus, low historical genetic diversity is not unusual among species for which ancient and historical samples have been analyzed. Nonetheless, the low koala mtDNA diversity does contrast with those of another Australian marsupial that went through a bottleneck, the northern hairy-nosed wombat. This wombat species lost half its diversity through a population bottleneck that was concurrent with the bottleneck that affected koalas; although the wombat went through a much greater proportional decline in population than the koala [22]. Thus, the continously low genetic diversity detected among koalas, Tasmanian devils and the Iberian lynx is not typical for all species. Some species examined across very long periods of time have demonstrated loss of genetic diversity as population sizes decreased, for example the Beringian steppe bison; while others tended to maintain their genetic diversity and population sizes across time, for example the woolly rhinoceros [23,24].

Koalas may have experienced population expansions and subsequent crashes repeatedly throughout the Quaternary, depending on the availability of eucalyptus, and, after human arrival, the intensity of human hunting. These more ancient bottlenecks may be responsible for the low diversity evident in our museum samples [17]. Koalas are currently threatened by co-infection with two lethal pathogens that are putting substantial pressure on the host species. The koala retrovirus (KoRV) is an oncogenic retrovirus that can cause high levels of mortality in captive and wild koala populations $[25,26]$. High titres of KoRV are correlated with infection by Chlamydia [27], which is now a major pathogen of wild koalas. Lack of genetic diversity may result in a general inability of the koala to resist either pathogen. Thus, biodiversity management strategies may have to consider prioritizing the preservation of KoRV-free populations given that genetic diversity may be low across the species.

The use of museum samples for genetic studies is not without difficulties. Besides the potential for a high failure rate, which proved to be over $50 \%$ in this study, the generation of error-free sequence is a crucial issue. Criteria have been suggested for authentication of ancient DNA sequences [28]. One of the suggested criteria is sub-cloning and sequencing of multiple clones. However, when a single sequence variant is expected, as with mtDNA haplotypes, it has recently been suggested that direct sequencing may be as accurate as cloning and sequencing [29]. We compared the results of high throughput sequencing of PCR amplicons to those of direct Sanger sequencing of PCR products. The high throughput sequencing data suggested that DNA damage affected only ca. $1 \%$ of the sites. Presumably Taq polymerase errors or nuclear copies of mtDNA (numts) also contributed to the sequence errors, although the effects of any numts could not be separately quantified [30]. Nonetheless, the consensus sequence of the reads was identical to the direct consensus Sanger sequencing results. Thus, under some circumstances, direct sequencing of 
ancient DNA may be adequate if steps are taken to confirm that reliable sequences can be obtained.

\section{Conclusions}

Koalas appear to have been characterized by low mtDNA diversity for at least the last 120 years. Contrary to expectations, historical diversity of the koala did not appear to differ from modern diversity and thus the most recent episode of overhunting likely played less of a role in the current paucity of mtDNA diversity than more ancient factors. Koala genetic diversity may have been affected by ancient climatic changes affecting their range or by previous aboriginal hunting pressure, before being impacted more recently by European Australian exploitation. The combined pressure on koalas has likely limited the accumulation of genetic diversity. When considering management strategies, it may be noted that low genetic diversity may not indicate recent inbreeding or founder events but may reflect a historical pattern for koalas. However, the long-term low diversity may have implications for the koala's ability to resist disease, as may be apparent from the spread of koala retrovirus and associated Chlamydia infections.

\section{Materials and methods \\ Samples}

Koala museum skin samples were generously provided by the Bohusläns Museum, Goteborg Museum, Kansas University of Kansas Natural History Museum, Museum of Comparative Zoology, Museum of Victoria, Queensland Museum, Royal Ontario Museum, Stockholm Museum, University of Michigan Museum of Zoology, and the Australian Museum (Table 1). Twenty-nine specimens from 28 individuals dating from the early 1870s to the early 1980s were sampled. When collection dates could not be constrained to a specific year, the range of dates possible for collection is listed.

\section{DNA extraction}

All extractions were performed in a dedicated ancient DNA facility in plexiglass PCR hoods, one hood dedicated to DNA extraction and a second hood to PCR setup. The room was never used for cell, molecular or genetic work on modern samples, and the Wildlife Diseases Department of the Leibniz-Institute for Zoo and Wildlife Research had never worked with koalas prior to the project. Personnel wore protective clothing to ensure that samples were not contaminated by the researchers during extraction or PCR setup. Approximately $250 \mathrm{mg}$ of skin tissue was extracted using the Geneclean Ancient DNA Extraction Kit from MP Biomedicals, USA. The protocol of the kit was followed according to the manufacturer's instructions; the same kit had previously been used successfully for ancient DNA extraction from samples of rat skin and woolly mammoth bone [31,32]. Mock extractions were performed with each set of koala museum specimens as a control for contamination during the extraction procedure. Subsequent to each extraction, the DNA was further purified using QiaQuick spin columns (Qiagen) as described in Gilbert et al. [33].

\section{PCR and sequencing}

Primers were designed based on conserved regions in modern koala mtDNA. Primers targeted regions of the mitochondrial DNA control region found to have high variability in koalas according to Houlden et al. [10]. The primers were tested at the University of Illinois on modern koala DNA. Combinations tested were PCI-CRs1-F1 (5'- AGTACATTCATTTATTTACCACTAGCAT-3') or PCI-CRs1-F2 (5'-ATAGTACATTCATTTATTTACCAC TAGCA-3') with PCI-CRs1-R1 (5'- AAGATGATTTA TATGTAATTCTAGATACGC-3') to amplify a ca. 73 bp target (exclusive of primer lengths). PCI-CRs2-F1 (5-A CCAAATGCGTATCTAGAATTACA-3') was combined with PCI-CRs2-R1 (5'-TTTGCTTGGATTGGATATGCT-3') and PCI-CRs2-F2 (5'-TACCAAATGCGTATCTAGAAT TACA-3') with PCI-CRs2-R2 (5'-TTGCTTGGATTGGA TATGCT-3'). Primer sets that amplified short mtDNA regions were used in order to minimize negative results associated with DNA damage in museum samples. Both PCI-CRs2 primer combinations target the same $112 \mathrm{bp}$ (without primers) region. The two reverse primers of the PCI-CRs1 amplicon overlaps with the two forward primers of the PCI-CRs2 amplicon and thus, the region sequenced did not overlap. A combination of the two sets was also used (PCI-CRs1-F1/PCI-CRs2-R1) to obtain a larger product $(\sim 201 \mathrm{bp})$ encompassing both the 73 and $112 \mathrm{bp}$ amplicons including the region of primer overlap between the smaller amplicons. The primers were tested with a dilution series of koala DNA to mimic the scarcity of DNA present in ancient samples. Each of the selected primers and combinations except PCI-CRs1-F1/ PCICRs2-R1 worked under this test regime on the lowest koala DNA dilution.

For all the samples, multiple independent PCRs were performed for each extract, and multiple extracts were obtained from each specimen. For all samples positive PCRs were replicated at least twice per primer set to avoid overestimation of diversity associated with ancient DNA damage. All amplifications from museum specimens were performed in a total volume of $34 \mu \mathrm{l}$ using $5.5 \mu \mathrm{l}$ of sample extract, $200 \mathrm{nM}$ of primers, and $0.5 \mathrm{U}$ Platinum HiFi supermix (Invitrogen). Cycling conditions were: $94^{\circ} \mathrm{C}$ for $4 \mathrm{~min}$; 60 cycles at $94^{\circ} \mathrm{C}$ for $30 \mathrm{~s}, 55^{\circ} \mathrm{C}$ for $30 \mathrm{~s}, 72^{\circ} \mathrm{C}$ for $30 \mathrm{~s}$; and at $72^{\circ} \mathrm{C}$ for $10 \mathrm{~min}$, with the samples then held at $4^{\circ} \mathrm{C}$ [31]. PCR products were visualized on a $3 \%$ agarose gel using GelRed nucleic acid gel stain 
by Biotium. PCR products were purified using the Qiaquick PCR clean up kit (Qiagen).

The positive samples were amplified in duplicate or triplicate to control for DNA damage induced errors that almost universally characterize ancient or degraded DNA samples. A subset of 8 samples from 7 individual koalas, representing the range of collection dates that provided positive results, was selected for GS FLX sequencing using a specific multiplex identifier (MID) according to manufacturer's instructions (Roche Life Sciences) (Table 1). The sequencing coverage depth obtained using this method effectively substitutes for the laborious cloning steps usually applied in ancient DNA studies and provides equivalent information with much higher sequence coverage [34]. Analyses of the GS FLX sequences for these seven samples indicated that only a very low degree of DNA damage was present (Figure 1). For each individual, every position along the sequences was examined for variation among reads, with the consensus character state (present at a frequency $>97 \%$ in every case) recorded for each nucleotide site for each individual. The proportion of sequences that had any of the three non-consensus character states was also recorded. Across the length of the sequence, there are 12 types of observable base errors, since for each of the 4 consensus character states there are three potential non-consensus character states, each representing a type of error (Figure 1). The percentage of reads with each type of error was averaged across the length of the sequence; for example the proportion of $\mathrm{C}$ to $\mathrm{T}$ changes was calculated by examining all positions at which a cytosine was the consensus character state. At all of these positions, the proportion of reads with a thymine was recorded, with the average rate of $\mathrm{C}$ - $\mathrm{T}$ error estimated by calculating, across all nucleotide sites at which the consensus was cytosine, the proportion of all reads with thymine. For six out of seven koalas, each of the 12 possible base errors comprised less than $1 \%$ of the total reads across all nucleotide positions. One koala, Pci-maex1738, had an elevated C-T error rate (Figure 1). However, for this individual, several positions with cytosine had relatively low GS FLX read coverage, and the higher error estimate was due to lower read coverage depth at a few positions. Thus, this was a sequence sampling effect rather than actual increased damage. Even after discounting these artifacts, C-T and G-A transitions were more frequent than other apparent base changes (Figure 1), consistent with the damage profiles expected for ancient DNA [35].

Given that DNA damage induced change appeared to be low across the koala museum specimens, we hypothesized that direct Sanger sequencing would be as effective for sequencing as the GS FLX method. Four of the seven high throughput sequenced koala samples were Sanger sequenced directly (Pci-MCZ 12454,
Pci-c2831, Pci-um3435, and Pci-QM J6480) to allow for comparison of the two methods; among them were two of the koalas that showed a higher percentage of damage induced change for certain substitutions (Pci-c2831 and Pci-QM J6480 Figure 1 for example). The direct Sanger method generated sequences identical to those of consensus sequence of GS FLX for all four samples. In two individuals, a single base conflict between the first and second amplicon sequence result was resolved by a third direct sequencing. However, none of the museum koala samples indicated multiple co-occurring sequences or unreadable bases were present. Based on the consistency between direct and high throughput sequencing results, the remaining samples were directly Sanger sequenced. Haplotype sequences are included in the Additional Files 1 and 2.

Of the 3 samples that were successfully amplified with only a single primer set, only Pci-6121 yielded a koala sequence. The haplotype based on this single fragment was not novel. Since only one mtDNA fragment was retrieved from Pci-6121 and it was identical to K5 haplotype it was not further analyzed (Figure 2).

\section{GS FLX data DNA damage estimation}

GS FLX sequence reads were first sorted by MID and . fna and .qual files were transformed into fastq format using perl scripts. Primer regions were removed from the analysis by trimming both ends of each read, and only fragments $36 \mathrm{bp}$ and longer were considered for mapping assembly. Each one of the seven datasets was then mapped to a reference corresponding to the $P$. cinereus mitochondrial control region using BWA (Version 0.5.9) [36]. The following GenBank mitochondrial control region references were used for sequence mapping, i.e., AJ005848, AJ005847, and AJ012063. The reference sequences were chosen based on haplotype similarity. Alignments were then converted to pileup format (samtools 0.1.17) [37] and a perl script was designed to estimate damage by extracting and sorting all the substitutions present at each position in the alignment. The algorithm generated per-position variation and summarized data, which allowed the percentage determination of damage patterns.

\section{Alignments, minimum spanning networks, and rarefaction analysis}

Alignment and comparison of the archival sample data with modern sequences were performed using Clustal $\mathrm{X}$ and Bioedit, respectively. The modern koala sequences were obtained from Genbank (accession numbers AJ005846AJ005863, AJ012057-AJ012064). Minimum spanning networks of the haplotypes were generated using TCS 1.21 
software at the default 95\% connection limit [38]. Rarefaction analysis was performed using Analytic Rarefaction 2.0 software that uses Tippers [39] equations to estimate the number of haplotypes. As input we used Houldens et al. [8] 208 koala dataset and analysis was performed following the developers instructions [40]. Plotting of the results was performed using Microsoft Excel 2010.

\section{Additional files}

Additional file 1: FASTA formatted files of the consensus haplotype
sequences for the $73 \mathrm{bp}$ and 112 bp museum koala amplicons.
Additional file 2: FASTA formatted files of the consensus haplotype
sequences for the $73 \mathrm{bp}$ and 112 bp museum koala amplicons.

\section{Competing interests}

The authors declare no competing interests.

\begin{abstract}
Author contributions
$K T$ performed ancient DNA extractions, PCR and sequencing experiments. YI performed all modern koala DNA work. KT and MCAA performed bioinformatics and phylogenetic analyses for the study. KMH provided koala samples and curatorial information and wrote parts of the manuscript. KT, ALR and ADG designed the study, contributed to the experimental work and analysis and wrote the manuscript. All authors read and approved the final manuscript.
\end{abstract}

\section{Acknowledgments}

The authors wish to thank S. Calvignac-Spencer for help in the initial stages of this project and Karin Hönig for technical assistance. We also thank Sergios-Orestis Kolokotronis and Simon Y.W. Ho for helpful suggestions. We thank G. Gordon, K. Aplin, and S. Jackson for helpful discussions. The authors thank Lauren Helgen and Paige Engelbrektsson for assistance with the koala distribution map. For museum specimens, we thank F. Johansson and G. Nilson (Bohusläns Museum), R. Timm (Natural History Museum - University of Kansas), J. Chupasko and H. Hoekstra (Harvard Museum of Comparative Zoology), W. Longmore (Victoria Museum, O. Grönwall and U. Johansson (Swedish Natural History Museum), J. Eger (Royal Ontario Museum), S. Hinshaw (University of Michigan Museum of Zoology), D. Stemmer and C. Kemper (South Australian Museum), S. Ingleby (Australian Museum), S. Van Dyck and H. Janetzki (Queensland Museum). For modern koala samples, we thank R. Hanson, M. Malasky, M. Bush, J. Graves, D. Wildt, W. Sherwin and S. O'Brien. We also thank the San Diego Zoo, Columbus Zoo and San Francisco Zoo for samples. M.C.A.A. was supported by The Australian Research Council, Lundbeck Foundation 'Pathogen Palaeogenomes', and the Danish National Research Foundation 'GeoGenetics' grants. K.M.H was supported by the Smithsonian Institution. This research was supported by Grant Number R01GM092706 from the National Institute of General Medical Sciences. The content is solely the responsibility of the authors and does not necessarily represent the official views of the National Institute of General Medical Sciences or the National Institutes of Health.

\section{Author details}

'Leibniz-Institute for Zoo and Wildlife Research, Berlin 10315, Germany.

${ }^{2}$ GeoGenetics, Natural History Museum of Denmark, University of Copenhagen, Østervoldgade 5-7, Copenhagen, DK 1350, Denmark. ${ }^{3}$ Department of Animal Sciences, University of Illinois at Urbana-Champaign, Urbana, IL 61801, USA. ${ }^{4}$ Smithsonian Institution, National Museum of Natural History, Washington, DC 20560-0108, USA.

Received: 24 May 2012 Accepted: 27 September 2012 Published: 24 October 2012

\section{References}

1. Jackson SM: Koala: origins of an icon: Allen \& Unwin; 2007.

2. O'Brien SJ: Genetic and Phylogenetic Analyses of Endangered Species. Annu Rev Genet 1994, 28(1):467-489.
3. Taylor AC, Graves JA, Murray ND, Sherwin WB: Conservation genetics of the koala (Phascolarctos cinereus). II. Limited variability in minisatellite DNA sequences. Biochem Genet 1991, 29(7-8):355-363.

4. Cristescu R, Sherwin W, Handasyde K, Cahill V, Cooper D: Detecting bottlenecks using BOTTLENECK 1.2.02 in wild populations: the importance of the microsatellite structure. Conserv Genet 2010, 11(3):1043-1049.

5. Leonard JA, Vila C, Wayne RK: Legacy lost: genetic variability and population size of extirpated US grey wolves (Canis lupus). Mol Ecol 2005, 14(1):9-17.

6. Whitehouse AM, Harley EH: Post-bottleneck genetic diversity of elephant populations in South Africa, revealed using microsatellite analysis. Mol Ecol 2001, 10(9):2139-2149.

7. Timms $\mathrm{P}$, Kato J, Maugeri $\mathrm{M}$, White $\mathrm{N}$ : DNA fingerprint analysis of a freerange koala population. Biochem Genet 1993, 31(9):363-374.

8. Houlden BA, England PR, Taylor AC, Greville WD, Sherwin WB: Low genetic variability of the koala Phascolarctos cinereus in south-eastern Australia following a severe population bottleneck. Mol Ecol 1996, 5(2):269-281.

9. Taylor AC, Graves JM, Murray ND, O'Brien SJ, Yuhki N, Sherwin B: Conservation genetics of the koala (Phascolarctos cinereus): low mitochondrial DNA variation amongst southern Australian populations. Genet Res 1997, 69(1):25-33.

10. Houlden BA, Costello BH, Sharkey D, Fowler EV, Melzer A, Ellis W, Carrick F, Baverstock PR, Elphinstone MS: Phylogeographic differentiation in the mitochondrial control region in the koala, Phascolarctos cinereus (Goldfuss 1817). Mol Ecol 1999, 8(6):999-1011.

11. Fowler EV, Houlden BA, Hoeben P, Timms P: Genetic diversity and gene flow among southeastern Queensland koalas (Phascolarctos cinereus). Mol Ecol 2000, 9(2):155-164.

12. Thomas WK, Paabo S, Villablanca FX, Wilson AC: Spatial and temporal continuity of kangaroo rat populations shown by sequencing mitochondrial DNA from museum specimens. J Mol Evol 1990, 31(2):101-112.

13. Campos PF, Willerslev E, Sher A, Orlando L, Axelsson E, Tikhonov A, Aaris-Sorensen K, Greenwood AD, Kahlke RD, Kosintsev P, et al: Ancient DNA analyses exclude humans as the driving force behind late Pleistocene musk ox (Ovibos moschatus) population dynamics. Proc Natl Acad Sci USA 2010, 107(12):5675-5680.

14. Miller W, Hayes VM, Ratan A, Petersen DC, Wittekindt NE, Miller J, Walenz B, Knight J, Qi J, Zhao F, et al: Genetic diversity and population structure of the endangered marsupial Sarcophilus harrisii (Tasmanian devil). Proc Natl Acad Sci USA 2011, 108(30):12348-12353.

15. Roberts RG, Flannery TF, Ayliffe LK, Yoshida H, Olley JM, Prideaux GJ, Laslett GM, Baynes A, Smith MA, Jones R, et al: New ages for the last Australian megafauna: Continent-wide extinction about 46,000 years ago. Science 2001, 292(5523):1888-1892.

16. Price GJ: Is the modern koala (Phascolarctos cinereus) a derived dwarf of a Pleistocene giant? Implications for testing megafauna extinction hypotheses. Quaternary Sci Rev 2008, 27(27-28):2516-2521.

17. Martin RW, Handasyde KA, Simpson S: The koala: natural history, conservation and management, Krieger Pub. Co. Sydney: University of New South Wales Press; 1999. distributed by Kriger Publishing Company, Malabar Florida.

18. Hawkins $C E$, Baars $C$, Hesterman H, Hocking GJ, Jones ME, Lazenby B, Mann D, Mooney N, Pemberton D, Pyecroft S, et al: Emerging disease and population decline of an island endemic, the Tasmanian devil Sarcophilus harrisii. Biol Conserv 2006, 131(2):307-324.

19. Miller W, Drautz DI, Janecka JE, Lesk AM, Ratan A, Tomsho LP, Packard M, Zhang Y, McClellan LR, Qi J, Zhao F, Gilbert MT, Dalén L, Arsuaga JL, Ericson PG, Huson DH, Helgen KM, Murphy WJ, Götherström A, Schuster SC: The mitochondrial genome sequence of the Tasmanian tiger (Thylacinus cynocephalus). Genome Res 2009, 19(2):213-20.

20. Menzies BR, Renfree MB, Heider T, Mayer F, Hildebrandt TB, Pask AJ: Limited genetic diversity preceded extinction of the Tasmanian tiger. PLoS One 2012, 7(4):e35433.

21. Rodriguez R, Ramirez O, Valdiosera CE, Garcia N, Alda F, Madurell-Malapeira J, Marmi J, Doadrio I, Willerslev E, Gotherstrom A, et al: 50,000 years of genetic uniformity in the critically endangered Iberian lynx. Mol Ecol 2011, 20(18):3785-3795.

22. Taylor AC, Sherwin WB, Wayne RK: Genetic variation of microsatellite loci in a bottlenecked species: the northern hairy-nosed wombat Lasiorhinus krefftii. Mol Ecol 1994, 3(4):277-290. 
23. Shapiro B, Drummond AJ, Rambaut A, Wilson MC, Matheus PE, Sher AV, Pybus OG, Gilbert MTP, Barnes I, Binladen J, et al: Rise and Fall of the Beringian Steppe Bison. Science 2004, 306(5701):1561-1565.

24. Lorenzen ED, Nogues-Bravo D, Orlando L, Weinstock J, Binladen J, Marske KA, Ugan A, Borregaard MK, Gilbert MTP, Nielsen R, et al: Species-specific responses of Late Quaternary megafauna to climate and humans. Nature 2011, 479(7373):359-364.

25. Hanger JJ, Bromham LD, McKee JJ, O'Brien TM, Robinson WF: The nucleotide sequence of koala (Phascolarctos cinereus) retrovirus: a novel type $C$ endogenous virus related to Gibbon ape leukemia virus. J Virol 2000, 74(9):4264-4272.

26. Tarlinton RE, Meers J, Young PR: Retroviral invasion of the koala genome. Nature 2006, 442(7098):79-81.

27. Tarlinton R, Meers J, Hanger J, Young P: Real-time reverse transcriptase PCR for the endogenous koala retrovirus reveals an association between plasma viral load and neoplastic disease in koalas. J Gen Virol 2005, 86(Pt 3):783-787.

28. Cooper A, Poinar HN: Ancient DNA: do it right or not at all. Science 2000, 289(5482):1139.

29. Winters M, Barta JL, Monroe C, Kemp BM: To clone or not to clone: method analysis for retrieving consensus sequences in ancient DNA samples. PLoS One 2011, 6(6):e21247.

30. Kolokotronis SO, Macphee RD, Greenwood AD: Detection of mitochondrial insertions in the nucleus (NuMts) of Pleistocene and modern muskoxen. BMC Evol Biol 2007, 7:67.

31. Wyatt KB, Campos PF, Gilbert MT, Kolokotronis SO, Hynes WH, DeSalle R, Ball SJ, Daszak P, MacPhee RD, Greenwood AD: Historical mammal extinction on Christmas Island (Indian Ocean) correlates with introduced infectious disease. PLoS One 2008, 3(11):e3602.

32. Roca AL, Ishida Y, Nikolaidis N, Kolokotronis SO, Fratpietro S, Stewardson K, Hensley S, Tisdale M, Boeskorov G, Greenwood AD: Genetic variation at hair length candidate genes in elephants and the extinct woolly mammoth. BMC Evol Biol 2009 9:232

33. Gilbert MT, Djurhuus D, Melchior L, Lynnerup N, Worobey M, Wilson AS, Andreasen C, Dissing J: mtDNA from hair and nail clarifies the genetic relationship of the 15th century Qilakitsoq Inuit mummies. Am J Phys Anthropol 2007, 133(2):847-853.

34. Binladen J, Gilbert MT, Bollback JP, Panitz F, Bendixen C, Nielsen R, Willerslev E: The use of coded PCR primers enables high-throughput sequencing of multiple homolog amplification products by 454 parallel sequencing. PLoS One 2007, 2(2):e197.

35. Willerslev E, Cooper A: Ancient DNA. Proc Biol Sci 2005, 272(1558):3-16.

36. Li H, Durbin R: Fast and accurate short read alignment with BurrowsWheeler transform. Bioinformatics 2009, 25(14):1754-1760.

37. Li H, Handsaker B, Wysoker A, Fennell T, Ruan J, Homer N, Marth G, Abecasis G, Durbin R, Genome Project Data Processing S: The Sequence Alignment/ Map format and SAMtools. Bioinformatics 2009, 25(16):2078-2079.

38. Clement M, Posada D, Crandall KA: TCS: a computer program to estimate gene genealogies. Mol Ecol 2000, 9(10):1657-1659.

39. Tipper JC: Rarefaction and rarefiction; the use and abuse of a method in paleoecology. Paleobiology 1979, 5(4):423-434.

40. Analytic Rarefaction 2.0: http://www.huntmountainsoftware.com/html/ rarefaction.html Copyright @ 2012 Hunt Mountain Software, LLC. All rights reserved.

\section{Submit your next manuscript to BioMed Central and take full advantage of:}

- Convenient online submission

- Thorough peer review

- No space constraints or color figure charges

- Immediate publication on acceptance

- Inclusion in PubMed, CAS, Scopus and Google Scholar

- Research which is freely available for redistribution

Submit your manuscript at www.biomedcentral.com/submit
C Biomed Central 